\title{
Asymmetric Route to Pyridines Bearing a Highly Functionalized 2-Alkyl Substituent by Aziridine Ring Opening Reactions
}

\author{
Giuseppe Alvaro, $\uparrow$ Romano Di Fabio, $\dagger$ Andrea Gualandi $\ddagger$ and Diego Savoia*, $\ddagger$ \\ $\dagger$ Psychiatric Centre of Excellence for Drug Discovery, GlaxoSmithKline S.p.A., via Fleming 4, 37135 Verona, \\ Italy \\ ¥ Dipartimento di Chimica "G. Ciamician", Università di Bologna, via Selmi 2, 40126 Bologna, Italy \\ * Corresponding author. Fax: +39-051-2099456; e-mail address: diego.savoia@ unibo.it
}

\section{List of content:}

General methods: S1

Analytical data for the aminoalchols 2a-i: S2-S7

Analytical data for the aminoalchols 3a-h: S7-S12

Analytical data for the primary amines: S12-S15

Analytical data for the benzamides 6b, 6f-i: S15-S17

Analytical data for the benzamides 7b, 7f-h: S18-S20

Analytical data for the diamine 8: S20

\section{General Methods}

Melting points are uncorrected. Optical rotations were measured on a digital polarimeter in a $1 \mathrm{dm}$ cell and $[\alpha]_{\mathrm{D}}$-values are given in $10^{-1} \mathrm{deg} \mathrm{cm}^{3} \mathrm{~g}^{-1} .{ }^{1} \mathrm{H}$ NMR spectra were recorded on 300 or 200 $\mathrm{MHz}$ instruments, for samples in $\mathrm{CDCl}_{3}$ which was stored over $\mathrm{Mg}:{ }^{1} \mathrm{H}$ chemical shifts are reported in ppm relative to $\mathrm{CHCl}_{3}\left(\delta_{\mathrm{H}}\right.$ 7.27) and $J$-values are given in. MS spectra were taken at an ionizing voltage of $70 \mathrm{eV}$ on a spectrometer equipped with GLC injection. LC-electron spray ionization (ESI) mass spectra were obtained with a single-quadrupole mass spectrometer. Chromatographic separations were performed on columns of $\mathrm{SiO}_{2}$ (230-400 mesh) or neutral aluminium oxide at medium pressure. Anhydrous THF, $\mathrm{CH}_{2} \mathrm{Cl}_{2}, \mathrm{CH}_{3} \mathrm{CN}$ were stored over molecular sieves. 


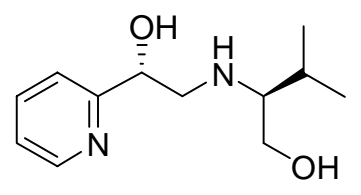

(S)-2-[(R)-2-Hydroxy-2-(2-pyridyl)ethylamino]-3-methyl-1-butanol (2a): Yellow oil; $[\alpha]_{D}^{20}$ $+24.2\left(c 1.3, \mathrm{CHCl}_{3}\right) . \mathrm{IR}$ (neat): $v=3406,2962,2925,1658,1597,1413,1260,1049,794,751 .{ }^{13} \mathrm{H}$ $\operatorname{NMR}\left(300 \mathrm{MHz}, \mathrm{CDCl}_{3}\right): \delta=8.55(\mathrm{ddd}, J=0.7, J=1.3, J=4.7,1 \mathrm{H}), 7.72(\mathrm{dt}, J=1.7, J=7.6,1$ $\mathrm{H}), 7.38(\mathrm{dt}, J=0.9, J=7.9,1 \mathrm{H}), 7.23(\mathrm{ddd}, J=0.7, J=4.7, J=7.6,1 \mathrm{H}), 4.86(\mathrm{dd}, J=3.6, J=$ $7.5,1 \mathrm{H}), 3.65(\mathrm{dd}, J=4.1, J=10.8,1 \mathrm{H}), 3.38(\mathrm{dd}, J=7.7, J=10.8,1 \mathrm{H}), 3.06(\mathrm{dd}, J=3.6, J=$ 12.0, $1 \mathrm{H}), 2.97(\mathrm{bs}, 3 \mathrm{H}), 2.90(\mathrm{dd}, J=7.5, J=12.0,1 \mathrm{H}), 2.43(\mathrm{ddd}, J=4.1, J=6.7, J=7.7,1 \mathrm{H})$, $1.81(\mathrm{sept}, J=6.9,1 \mathrm{H}), 0.97(\mathrm{~d}, J=6.8,3 \mathrm{H}), 0.90(\mathrm{~d}, J=6.8,6 \mathrm{H}) .{ }^{13} \mathrm{C} \mathrm{NMR}\left(75 \mathrm{MHz}, \mathrm{CDCl}_{3}\right)$ : $\delta=160.3,148.3,136.9,122.6,120.6,72.3,64.8,61.0,54.0,29.1,19.5,18.5$. GC-MS $m / z 207$ (2), 193 (26), 181(8), 175 (10), 121(44), 116 (60), 109 (100), 108 (24), 94 (25), 79 (19), 69 (11); MS (ES) $m / z 225.4(\mathrm{M}+\mathrm{H})^{+}$. Anal. Calcd for $\mathrm{C}_{12} \mathrm{H}_{20} \mathrm{~N}_{2} \mathrm{O}_{2}$ : C, 64.26; H, 8.99; N, 12.49. Found C, 64.06; H, 9.01; N, 12.44 .

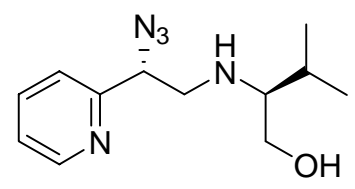

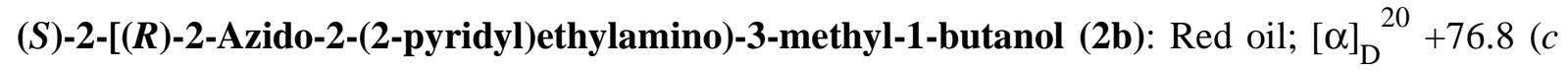
1.7, $\left.\mathrm{CHCl}_{3}\right) . \mathrm{IR}$ (neat): $v=3380,2956,2922,2102,1592,1472,1436,1155,1117 .{ }^{1} \mathrm{H}$ NMR $(300$ $\left.\mathrm{MHz}, \mathrm{CDCl}_{3}\right): \delta=8.61(\mathrm{ddd}, J=0.9, J=1.8, J=4.7,1 \mathrm{H}), 7.74(\mathrm{dt}, J=1.8, J=7.7,1 \mathrm{H}), 7.38(\mathrm{dt}$, $J=0.9, J=7.7,1 \mathrm{H}), 7.26(\mathrm{ddd}, J=1.1, J=4.7, J=7.5,1 \mathrm{H}), 4.64(\mathrm{dd}, J=5.0, J=7.6,1 \mathrm{H}), 3.59$ $(\mathrm{dd}, J=4.1, J=10.8,1 \mathrm{H}), 3.31(\mathrm{dd}, J=7.7, J=10.8,1 \mathrm{H}), 3.30(\mathrm{dd}, J=5.0, J=12.5,1 \mathrm{H}), 3.19$ $(\mathrm{dd}, J=7.6, J=12.5,1 \mathrm{H}), 2.39(\mathrm{dd}, J=4.1, J=7.7,1 \mathrm{H}), 2.14(\mathrm{bs}, 2 \mathrm{H}) 1.77(\mathrm{sept}, J=6.9,1 \mathrm{H})$, $0.94(\mathrm{~d}, J=6.9,3 \mathrm{H}), 0.88(\mathrm{~d}, J=6.9,3 \mathrm{H}) .{ }^{13} \mathrm{C} \mathrm{NMR}\left(75 \mathrm{MHz}, \mathrm{CDCl}_{3}\right): \delta=157.5,149.6,137.0$ 
123.2, 121.9, 66.7, 64.8, 61.0, 51.1, 29.2, 19.4, 18.4. MS (ES) $m / z 250.2(\mathrm{M}+\mathrm{H})^{+}, 272.1(\mathrm{M}+$ $\mathrm{Na})^{+}$. Anal. Calcd for $\mathrm{C}_{12} \mathrm{H}_{19} \mathrm{~N}_{5} \mathrm{O}: \mathrm{C}, 57.81 ; \mathrm{H}, 7.68 ; \mathrm{N}, 28.09 ; \mathrm{O}, 6.42$. Found $\mathrm{C}, 57.55 ; \mathrm{H}, 7.70$; 27.99 .

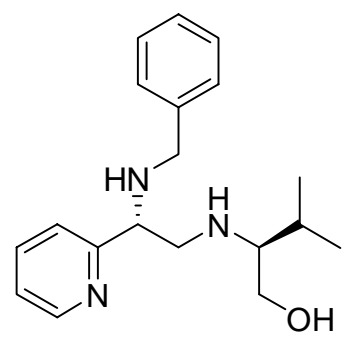

(S)-2-[(R)-2-(Benzylamino)-2-(2-pyridyl)ethylamino]-3-methy-1-lbutanol (2c): Yellow oil; $[\alpha]_{\mathrm{D}}{ }^{20}+30.8\left(c 1.7, \mathrm{CHCl}_{3}\right) ; \mathrm{IR}$ (neat): $v=3308,3061,3028,2956,2921,2864,1591,1570,1454$, 1434, 1366, 1121, 1048, 749, 699. ${ }^{1} \mathrm{H}$ NMR $\left(300 \mathrm{MHz}, \mathrm{CDCl}_{3}\right): \delta=8.63(\mathrm{ddd}, J=0.8, J=1.8, J=$ 4.8, $1 \mathrm{H}), 7.63(\mathrm{td}, J=1.8, J=7.7,1 \mathrm{H}), 7.35(\mathrm{~m}, 5 \mathrm{H}), 7.28(\mathrm{~m}, 1 \mathrm{H}), 7.23(\mathrm{ddd}, J=1.2, J=4.8, J$ $=7.5,1 \mathrm{H}), 3.87(\mathrm{dd}, J=5.9, J=7.2,1 \mathrm{H}), 3.72(\mathrm{~d}, J=12.9,1 \mathrm{H}), 3.61(\mathrm{~d}, J=12.9,1 \mathrm{H}), 3.59(\mathrm{dd}$, $J=4.0, J=10.7,1 \mathrm{H}), 3.35(\mathrm{dd}, J=7.8, J=10.7,1 \mathrm{H}), 2.91(\mathrm{~m}, 2 \mathrm{H}), 2.53(\mathrm{bs}, 3 \mathrm{H}), 2.39(\mathrm{dd}, J=$ $4.0, J=7.8,1 \mathrm{H}), 1.78(\mathrm{sept}, J=6.8,1 \mathrm{H}), 0.86(\mathrm{~d}, J=6.8,3 \mathrm{H}), 0.88(\mathrm{~d}, J=6.8,3 \mathrm{H}) .{ }^{13} \mathrm{C}$ NMR $\left(75 \mathrm{MHz}, \mathrm{CDCl}_{3}\right): \delta=161.7,149.5,140.1,136.4,128.3,128.2,126.9,122.7,122.3,64.6,63.2$, 52.8, 51.6, 29.4, 19.5, 18.7. MS (ES) $\mathrm{m} / z 314.3(\mathrm{M}+\mathrm{H})^{+}$. Anal. Calcd for $\mathrm{C}_{19} \mathrm{H}_{27} \mathrm{~N}_{3} \mathrm{O}: \mathrm{C}, 72.81$; H, 8.68; N, 13.41. Found C, 72.88; H, 8.71; N, 13.37.

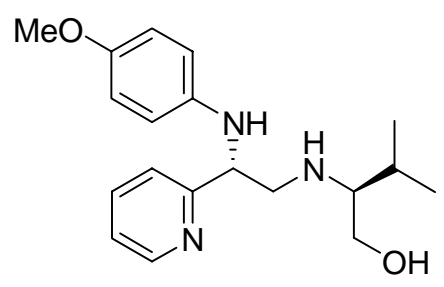

(S)-2-[(R)-2-(4-Methoxyphenylamino)-2-(2-pyridyl)ethylamino]-3-methyl-1-butanol (2d): Red oil; $[\alpha]_{\mathrm{D}}{ }^{20}+21.1\left(c 1.3, \mathrm{CHCl}_{3}\right)$. IR (neat): $v=3333,2957,2928,2872,2828,1592,1512,1465$, 
1435, 1239, 1039. ${ }^{13} \mathrm{H}$ NMR $\left(300 \mathrm{MHz}, \mathrm{CDCl}_{3}\right): \delta=8.58(\mathrm{ddd}, J=0.8, J=1.7, J=4.8,1 \mathrm{H}), 7.63$ $(\mathrm{dt}, J=1.7, J=7.6,1 \mathrm{H}), 7.36(\mathrm{dt}, J=0.8, J=7.8,1 \mathrm{H}), 7.18(\mathrm{ddd}, J=1.2, J=4.8, J=7.6,1 \mathrm{H})$, $6.72(\mathrm{~d}, J=9.1,2 \mathrm{H}), 6.60(\mathrm{~d}, J=9.1,2 \mathrm{H}), 4.54(\mathrm{dd}, J=4.8, J=7.1,1 \mathrm{H}), 3.78(\mathrm{~s}, 3 \mathrm{H}), 3.62(\mathrm{dd}$, $J=4.1, J=10.8,1 \mathrm{H}), 3.38(\mathrm{dd}, J=7.5, J=10.8,1 \mathrm{H}), 3.13(\mathrm{dd}, J=4.8, J=11.9,1 \mathrm{H}), 3.05(\mathrm{dd}, J$ $=7.1, J=11.9,1 \mathrm{H}), 2.73(\mathrm{bs}, 2 \mathrm{H}), 2.43(\mathrm{ddd}, J=4.1, J=6.5, J=7.5,1 \mathrm{H}), 1.81(\mathrm{sept}, J=6.8,1$ $\mathrm{H}), 0.93(\mathrm{~d}, J=6.8,3 \mathrm{H}), 0.87(\mathrm{~d}, J=6.8,6 \mathrm{H}) .{ }^{13} \mathrm{C} \mathrm{NMR}\left(75 \mathrm{MHz}, \mathrm{CDCl}_{3}\right): \delta=161.2,152.4$, $149.3,141.4,136.8,122.4,121.8,115.5,114.7,64.8,60.9,60.3,55.6,52.4,28.9,19.4,18.4 ; \mathrm{MS}$ (ES) $m / z 330.2(\mathrm{M}+\mathrm{H})^{+}$. Anal. Calcd for $\mathrm{C}_{19} \mathrm{H}_{27} \mathrm{~N}_{3} \mathrm{O}_{2}$ : C, 69.27; H, 8.26; N, 12.76. Found C, 69.52; H, 8.30; N, 12.71 .

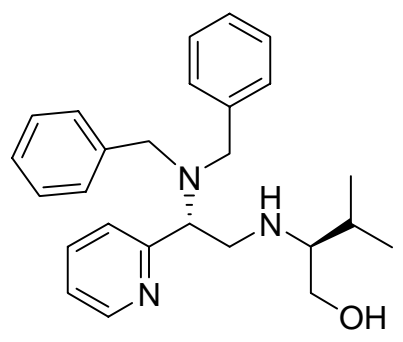

(S)-2-((R)-2-(dibenzylamino)-2-(pyridin-2-yl)ethylamino)-3-methylbutan-1-ol (2e): Red oil; $[\alpha]_{\mathrm{D}}{ }^{20}+24.3\left(c 1.4, \mathrm{CHCl}_{3}\right)$. IR (neat): $v=3357,3061,3028,2959,2925,1589,1453,1434,1261$, 1072, 1027, 698. ${ }^{1} \mathrm{H}$ NMR $\left(300 \mathrm{MHz}, \mathrm{CDCl}_{3}\right): \delta=8.60(\mathrm{ddd}, J=0.8, J=1.7, J=4.9,1 \mathrm{H}), 7.71$ $(\mathrm{td}, J=1.9, J=7.6,1 \mathrm{H}), 7.36(\mathrm{~m}, 9 \mathrm{H}), 7.23(\mathrm{~m}, 3 \mathrm{H}), 3.96(\mathrm{dd}, J=6.7, J=6.9,1 \mathrm{H}), 3.84(\mathrm{~d}, J=$ $13.82 \mathrm{H}), 3.64(\mathrm{dd}, J=4.1, J=10.8,1 \mathrm{H}), 3.42(\mathrm{~d}, J=13.82 \mathrm{H}), 3.37(\mathrm{~m}, 2 \mathrm{H}), 3.20(\mathrm{dd}, J=7.4, J$ $=11.6,1 \mathrm{H}), 2.38(\mathrm{dd}, J=4.1, J=6.9,1 \mathrm{H}), 1.76(\mathrm{sept}, J=6.8,1 \mathrm{H}), 0.95(\mathrm{~d}, J=6.8,3 \mathrm{H}), 0.89(\mathrm{~d}$, $J=6.8,6 \mathrm{H}) .{ }^{13} \mathrm{C}$ NMR $\left(75 \mathrm{MHz}, \mathrm{CDCl}_{3}\right) \delta=159.4,148.8,139.8,136.1,128.7,128.3,127.0$, $124.5,122.2,64.5,62.2,60.9,54.3,45.5,29.5,19.6,18.9 . \mathrm{MS}(\mathrm{ES}) \mathrm{m} / z$ 404.5 (M+H) ${ }^{+}$. Anal. Calcd for $\mathrm{C}_{26} \mathrm{H}_{33} \mathrm{~N}_{3} \mathrm{O}: \mathrm{C}, 77.38 ; \mathrm{H}, 8.24 ; \mathrm{N}, 10.41$. Found C, 77.10; H, 8.27; N, 10.37 . 


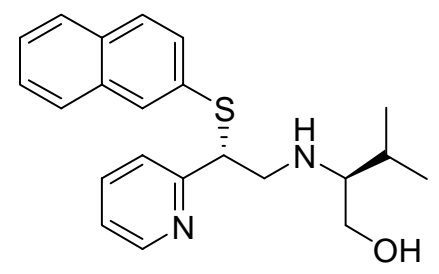

(S)-2-[(R)-2-(2-Naphthylthio)-2-(2-pyridyl)ethylamino]-3-methyl-1-butanol (2f): Yellowish oil;

$[\alpha]_{\mathrm{D}}{ }^{20}+51.8\left(c 1.7, \mathrm{CHCl}_{3}\right) . \mathrm{IR}($ neat) $: v=3412,3330,3055,3009,2954,1584,1565,1474,1432$, 1105, 1044, 748, 692. ${ }^{1} \mathrm{H}$ NMR $\left(300 \mathrm{MHz}, \mathrm{CDCl}_{3}\right): \delta=8.56(\mathrm{ddd}, J=0.9, J=1.9, J=4.8,1 \mathrm{H})$, $7.71(\mathrm{~m}, 2 \mathrm{H}), 7.69(\mathrm{~m}, 2 \mathrm{H}), 7.59(\mathrm{dt}, J=1.8, J=7.7,1 \mathrm{H}), 7.46(\mathrm{~m}, 2 \mathrm{H}), 7.39(\mathrm{dd}, J=1.8, J=$ $8.5,1 \mathrm{H}), 7.28(\mathrm{dt}, J=0.9, J=7.8,1 \mathrm{H}), 7.16(\mathrm{ddd}, J=1.1, J=4.9, J=7.8,1 \mathrm{H}), 4.61(\mathrm{dd}, J=6.4$, $J=6.6,1 \mathrm{H}), 3.60(\mathrm{dd}, J=4.1, J=10.8,1 \mathrm{H}), 3.40(\mathrm{dd}, J=6.4, J=12.3,1 \mathrm{H}), 3.33(\mathrm{dd}, J=7.4, J$ $=10.8,1 \mathrm{H}), 3.29(\mathrm{dd}, J=6.4, J=12.2,1 \mathrm{H}), 2.65(\mathrm{bs}, 2 \mathrm{H}), 2.42(\mathrm{ddd}, J=4.1, J=6.4, J=7.4,1$ $\mathrm{H}), 1.78(\mathrm{sept}, J=6.8,1 \mathrm{H}), 0.93(\mathrm{~d}, J=6.8,3 \mathrm{H}), 0.88(\mathrm{~d}, J=6.8,3 \mathrm{H}) .{ }^{13} \mathrm{C} \mathrm{NMR}(75 \mathrm{MHz}$, $\left.\mathrm{CDCl}_{3}\right): \delta=159.8,149.4,136.6,133.5,132.3,131.4,130.9,129.4,128.4,127.6,127.4,126.5$, $126.2,123.1,122.4,64.7,60.8,54.8,50.5,29.2,19.6,18.6 . \mathrm{MS}(\mathrm{ES}) \mathrm{m} / z 367.3(\mathrm{M}+\mathrm{H})^{+}, 389.1(\mathrm{M}$ $+\mathrm{Na})^{+}$. Anal. Calcd for $\mathrm{C}_{22} \mathrm{H}_{26} \mathrm{~N}_{2} \mathrm{OS}$ : C, 72.09; H, 7.15; N, 7.64. Found C, 71.83; H, 7.16; N, 7.62.<smiles>CC(C)[C@H](CO)NCC(Sc1ccccc1)c1ccccn1</smiles>

(S)-2-[(R)-2-(Phenylthio)-2-(2-pyridyl)ethylamino]-3-methyl-1-butanol (2g): Yellow oil; $[\alpha]_{\mathrm{D}}^{20}$ +87.7 (c 1.2, $\left.\mathrm{CHCl}_{3}\right)$. IR (neat): $v=3419,3332,3056,3006,2957,2925,2871,1589,1569,1471$, $1434,1385,1109,1049,1025,747,691 .{ }^{1} \mathrm{H}$ NMR $\left(300 \mathrm{MHz}, \mathrm{CDCl}_{3}\right): \delta=8.57(\mathrm{ddd}, J=0.9, J=$ $1.8, J=4.8,1 \mathrm{H}), 7.74(\mathrm{dt}, J=1.8, J=7.7,1 \mathrm{H}), 7.33(\mathrm{~m}, 3 \mathrm{H}), 7.25(\mathrm{~m}, 3 \mathrm{H}), 7.17(\mathrm{ddd}, J=0.9, J$ $=4.8, J=7.4,1 \mathrm{H}), 4.47(\mathrm{dd}, J=6.7, J=6.9,1 \mathrm{H}), 3.60(\mathrm{dd}, J=4.1, J=10.7,1 \mathrm{H}), 3.35(\mathrm{dd}, J=$ 
$6.7, J=12.3,1 \mathrm{H}), 3.31(\mathrm{dd}, J=7.6, J=10.7,1 \mathrm{H}), 3.26(\mathrm{dd}, J=6.9, J=12.3,1 \mathrm{H}), 2.48(\mathrm{bs}, 2 \mathrm{H})$, $2.31(\mathrm{ddd}, J=4.1, J=7.1, J=7.6,1 \mathrm{H}), 1.77(\mathrm{sept}, J=6.9,1 \mathrm{H}), 0.93(\mathrm{~d}, J=6.8,3 \mathrm{H}), 0.89(\mathrm{~d}, J=$ $6.8,6 \mathrm{H}) .{ }^{13} \mathrm{C} \mathrm{NMR}\left(75 \mathrm{MHz}, \mathrm{CDCl}_{3}\right): \delta=159.9,149.3,136.5,134.1,132.2,128.8,127.3,123.0$, 122.2, 64.5, 60.8, 55.2, 50.6, 29.2, 19.5, 18.6. MS (ES) $m / z 317.1(\mathrm{M}+\mathrm{H})^{+}$. Anal. Calcd for $\mathrm{C}_{17} \mathrm{H}_{23} \mathrm{~N}_{2} \mathrm{OS}: \mathrm{C}, 67.29 ; \mathrm{H}, 7.64 ; \mathrm{N}, 9.23$. Found C, 67.01; H, 7.65; N, 9.21.

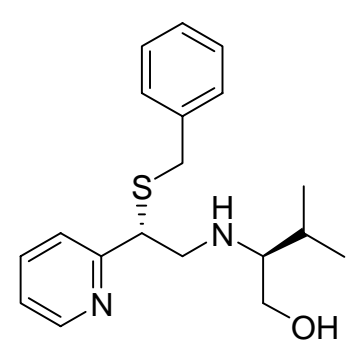

(S)-2-[(R)-2-(Benzylthio)-2-(2-pyridyl)ethylamino]-3-methyl-1-butanol (2h): Yellow oil; $[\alpha]_{\mathrm{D}}{ }^{20}$ $+143.0\left(c 1.2, \mathrm{CHCl}_{3}\right)$. IR (neat): $v=3383,3061,3028,2956,2925,1590,1494,1471,1453,1434$ 1049, 749, 702. ${ }^{1} \mathrm{H} \mathrm{NMR}\left(300 \mathrm{MHz}, \mathrm{CDCl}_{3}\right): \delta=8.56(\mathrm{ddd}, J=0.8, J=1.6, J=4.8,1 \mathrm{H}), 7.74(\mathrm{dt}$, $J=1.8, J=7.8,1 \mathrm{H}), 7.29(\mathrm{~m}, 5 \mathrm{H}), 7.18(\mathrm{~m}, 2 \mathrm{H}), 3.97(\mathrm{dd}, J=6.8, J=6.8,1 \mathrm{H}), 3.79(\mathrm{~d}, J=13.3$, $1 \mathrm{H}), 3.60(\mathrm{~d}, J=13.3,1 \mathrm{H}), 3.54(\mathrm{dd}, J=4.1, J=10.7,1 \mathrm{H}), 3.24(\mathrm{dd}, J=7.6, J=10.7,1 \mathrm{H}), 3.21$ $(\mathrm{dd}, J=6.7, J=12.1,1 \mathrm{H}), 3.11(\mathrm{dd}, J=7.0, J=12.1,1 \mathrm{H}), 2.40(\mathrm{bs}, 2 \mathrm{H}), 2.31(\mathrm{dd}, J=4.1, J=$ 7.6, $1 \mathrm{H}), 1.71(\mathrm{sept}, J=6.9,1 \mathrm{H}), 0.89(\mathrm{~d}, J=6.8,3 \mathrm{H}), 0.84(\mathrm{~d}, J=6.8,6 \mathrm{H}) .{ }^{13} \mathrm{C}$ NMR $(75$ $\left.\mathrm{MHz}, \mathrm{CDCl}_{3}\right): \delta=160.6,149.3,137.9,136.7,128.9,128.5,127.0,122.9,122.2,64.5,60.8,51.2$ 50.6, 35.4, 29.2, 19.5, 18.6. GC-MS m/z 105 (100), 91 (93), 93 (70), 104 (40), 78 (31), 65 (30), 226 (26), 124 (26), 194 (25), 226 (18), 207 (16), 51 (16), 136 (12). Anal. Calcd for $\mathrm{C}_{19} \mathrm{H}_{26} \mathrm{~N}_{2} \mathrm{OS}$ : C, 69.05; H, 7.93; N, 8.48. Found C, 69.16; H, 7.95; N, 8.46. 


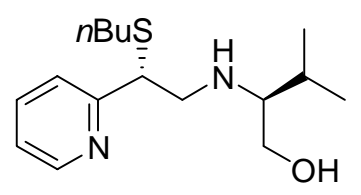

(S)-2-[(R)-2-(Butylthio)-2-(2-pyridyl)ethylamino]-3-methyl-1-butanol (2i): Yellow oil; $[\alpha]_{D}^{20}$ $+36.8\left(c 1.0, \mathrm{CHCl}_{3}\right) . \mathrm{IR}$ (neat): $v=3346,2957,2926,2872,1590,1569,1468,1434,1049,788$, 748. ${ }^{1} \mathrm{H}$ NMR $\left(300 \mathrm{MHz}, \mathrm{CDCl}_{3}\right): \delta=8.54(\mathrm{ddd}, J=0.8, J=1.8, J=4.7,1 \mathrm{H}), 7.67(\mathrm{dt}, J=1.8, J$ $=7.7,1 \mathrm{H}), 7.39(\mathrm{dt}, J=0.8, J=7.7,1 \mathrm{H}), 7.17(\mathrm{ddd}, J=1.0, J=4.7, J=7.7,1 \mathrm{H}), 4.06(\mathrm{dd}, J=$ $7.1, J=7.1,1 \mathrm{H}), 3.59(\mathrm{dd}, J=4.2, J=10.7,1 \mathrm{H}), 3.30(\mathrm{dd}, J=7.5, J=10.7,1 \mathrm{H}), 3.24(\mathrm{dd}, J=$ $7.1, J=12.1,1 \mathrm{H}), 3.16(\mathrm{dd}, J=7.1, J=12.1,1 \mathrm{H}), 2.60(\mathrm{bs}, 2 \mathrm{H}), 2.43(\mathrm{dd}, J=6.8, J=7.2,2 \mathrm{H})$, $2.39(\mathrm{dd}, J=4.2, J=7.5,1 \mathrm{H}), 1.78(\mathrm{sept}, J=6.9,1 \mathrm{H}), 1.47(\mathrm{~m}, 2 \mathrm{H}), 1.31(\mathrm{~m}, 2 \mathrm{H}), 0.92(\mathrm{~d}, J=$ $6.9,3 \mathrm{H}), 0.88(\mathrm{~d}, J=6.9,3 \mathrm{H}), 0.84(\mathrm{t}, J=7.3,3 \mathrm{H}) .{ }^{13} \mathrm{C} \mathrm{NMR}\left(75 \mathrm{MHz}, \mathrm{CDCl}_{3}\right): \delta=160.9$, $149.1,136.7,122.8,122.1,64.6,60.8,51.6,50.6,31.6,30.4,29.2,21.9,19.5,18.6,13.6 . \mathrm{MS}(\mathrm{ES})$ $m / z 297.2(\mathrm{M}+\mathrm{H})^{+}$. Anal. Calcd for $\mathrm{C}_{16} \mathrm{H}_{28} \mathrm{~N}_{2} \mathrm{OS}: \mathrm{C}, 64.82 ; \mathrm{H}, 9.52 ; \mathrm{N}, 9.45$. Found $\mathrm{C}, 64.73 ; \mathrm{H}$, $9.52 ; \mathrm{N}, 9.43$.

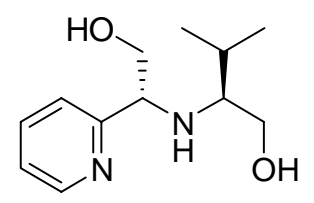

(S)-2-[(R)-2-Hydroxy-1-(2-pyridyl)ethylamino]-3-methyl-1-butanol (3a): Yellow oil; $[\alpha]_{\mathrm{D}}^{20}$ $+38.6\left(c 1.2, \mathrm{CHCl}_{3}\right) . \mathrm{IR}$ (neat) $v=3330,2957,2921,2873,1670,1595,1571,1468,1436,1049$, 774, 751. ${ }^{1} \mathrm{H}$ NMR $\left(300 \mathrm{MHz}, \mathrm{CDCl}_{3}\right): \delta=8.60(\mathrm{ddd}, J=0.8, J=1.6, J=4.9,1 \mathrm{H}), 7.75(\mathrm{dt}, J=$ $1.8, J=7.7,1 \mathrm{H}), 7.34(\mathrm{dt}, J=0.9, J=7.7,1 \mathrm{H}), 7.28(\mathrm{~m}, 1 \mathrm{H}), 4.12(\mathrm{dd}, J=4.2, J=6.6,1 \mathrm{H}), 3.96$ $(\mathrm{dd}, J=4.2, J=11.3,1 \mathrm{H}), 3.84(\mathrm{dd}, J=6.6, J=11.3,1 \mathrm{H}), 3.81(\mathrm{dd}, J=3.7, J=11.6,1 \mathrm{H}), 3.65$ $(\mathrm{dd}, J=6.1, J=11.6,1 \mathrm{H}), 2.47(\mathrm{dd}, J=3.7, J=6.1,1 \mathrm{H}), 1.81(\mathrm{sept}, J=6.8,1 \mathrm{H}), 0.95(\mathrm{~d}, J=6.8$ $3 \mathrm{H}), 0.90(\mathrm{~d}, J=6.8,6 \mathrm{H}) .{ }^{13} \mathrm{C}$ NMR $\left(75 \mathrm{MHz}, \mathrm{CDCl}_{3}\right) \delta=159.7,149.1,137.1,123.1,122.7,65.6$, 
64.1, 62.8, 61.1, 29.3, 19.3, 18.9. MS (ES) $\mathrm{m} / z 225.4(\mathrm{M}+\mathrm{H})^{+}$. Anal. Calcd for $\mathrm{C}_{12} \mathrm{H}_{20} \mathrm{~N}_{2} \mathrm{O}_{2}$ : C, 64.26; H, 8.99; N, 12.49. Found C, 64.10; H, 9.02; N, 12.45.

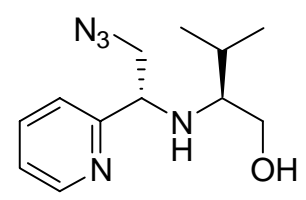

(S)-2-[(S)-2-Azido-1-(2-pyridyl)ethylamino]-3-methyl-1-butanol (3b): Yellow oil; $[\alpha]_{\mathrm{D}}{ }^{20}+52.8$ $\left(c 1.1, \mathrm{CHCl}_{3}\right) . \mathrm{IR}$ (neat): $v=3378,2950,2923,2106,1594,1470,1438,1153,1112 .{ }^{1} \mathrm{H}$ NMR $\left(300 \mathrm{MHz}, \mathrm{CDCl}_{3}\right): \delta=8.64(\mathrm{ddd}, J=0.9, J=1.7, J=4.8,1 \mathrm{H}), 7.72(\mathrm{dt}, J=1.8, J=7.7,1 \mathrm{H})$, $7.30(\mathrm{dt}, J=0.9, J=7.7,1 \mathrm{H}), 7.26(\mathrm{ddd}, J=0.9, J=4.8, J=7.7,1 \mathrm{H}), 3.98(\mathrm{dd}, J=6.5, J=6.5,1$ H), $3.69(\mathrm{dd}, J=3.9, J=11.1,1 \mathrm{H}), 3.63(\mathrm{dd}, J=6.5, J=12.1,1 \mathrm{H}), 3.55(\mathrm{dd}, J=6.57 .1, J=12.1$, $1 \mathrm{H}), 3.50(\mathrm{dd}, J=5.4, J=11.1,1 \mathrm{H}), 2.54(\mathrm{bs}, 2 \mathrm{H}), 2.31(\mathrm{dd}, J=3.9, J=5.4,1 \mathrm{H}), 1.69(\mathrm{sept}, J=$ $6.8,1 \mathrm{H}), 0.86(\mathrm{~d}, J=6.8,3 \mathrm{H}), 0.82(\mathrm{~d}, J=6.8,3 \mathrm{H}) .{ }^{13} \mathrm{C} \mathrm{NMR}\left(75 \mathrm{MHz}, \mathrm{CDCl}_{3}\right): \delta=161.7$, 149.7, 136.6, 122.9, 122.7, 63.2, 31.6, 30.9, 29.5, 19.4, 18.7. MS (ES) $m / z 250.2(\mathrm{M}+\mathrm{H})^{+}$. Anal. Calcd for $\mathrm{C}_{12} \mathrm{H}_{19} \mathrm{~N}_{5} \mathrm{O}: \mathrm{C}, 57.81 ; \mathrm{H}, 7.68 ; \mathrm{N}, 28.09 ; \mathrm{O}, 6.42$. Found $\mathrm{C}, 57.58 ; \mathrm{H}, 7.71 ; 28.00$.

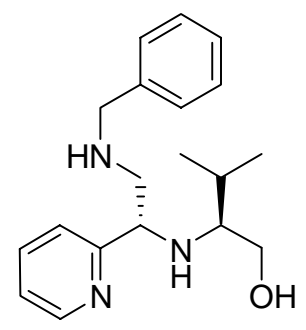

$(S)-2-[(S)-2-B e n z y l a m i n o-1-(2-p y r i d y l) e t h y l a m i n o]-3-m e t h y l-1-b u t a n o l ~(3 c): ~ Y e l l o w ~ o i l . ~[\alpha]_{D}{ }^{20}$ $+22.3\left(c 1.7, \mathrm{CHCl}_{3}\right)$. IR (neat): $v=3308,3062,2955,2915,2866,2850,1590,1454,1434,1366$, 1120, 749, 698. ${ }^{1} \mathrm{H}$ NMR $\left(300 \mathrm{MHz}, \mathrm{CDCl}_{3}\right): \delta=8.60(\mathrm{ddd}, J=0.8, J=1.6, J=4.8,1 \mathrm{H}), 7.68(\mathrm{dt}$, $J=1.8, J=7.6,1 \mathrm{H}), 7.32(\mathrm{~m}, 5 \mathrm{H}), 7.15(\mathrm{~m}, 1 \mathrm{H}), 7.18(\mathrm{ddd}, J=0.8, J=4.8, J=7.6,1 \mathrm{H}), 3.91$ $(\mathrm{dd}, J=4.6, J=8.5,1 \mathrm{H}), 3.69(\mathrm{~d}, J=12.7,1 \mathrm{H}), 3.64(\mathrm{dd}, J=4.6, J=11.1,1 \mathrm{H}), 3.63(\mathrm{~d}, J=12.7$, 
$1 \mathrm{H}), 3.42(\mathrm{dd}, J=7.7, J=11.1,1 \mathrm{H}), 3.03(\mathrm{dd}, J=4.4, J=12.0,1 \mathrm{H}), 2.90(\mathrm{dd}, J=8.6, J=12.0,1$ H), 2.67 (bs, $3 \mathrm{H}), 2.43(\mathrm{dd}, J=4.6, J=7.7,1 \mathrm{H}), 1.82(\mathrm{sept}, J=6.9,1 \mathrm{H}), 0.97(\mathrm{~d}, J=6.9,3 \mathrm{H})$, $0.91(\mathrm{~d}, J=6.9,3 \mathrm{H}) .{ }^{13} \mathrm{C} \mathrm{NMR}\left(75 \mathrm{MHz}, \mathrm{CDCl}_{3}\right): \delta=161.9,149.2,140.1,136.4,128.3,128.2$, $127.1,127.1,126.7,122.7,122.3,64.7,62.5,61.7,60.3,54.5,53.6,30.2,19.3,18.7 . \mathrm{MS}(\mathrm{ES}) \mathrm{m} / \mathrm{z}$ $314.3(\mathrm{M}+\mathrm{H})^{+}$. Anal. Calcd for $\mathrm{C}_{19} \mathrm{H}_{27} \mathrm{~N}_{3} \mathrm{O}: \mathrm{C}, 72.81 ; \mathrm{H}, 8.68 ; \mathrm{N}, 13.41$. Found C, 73,15; H, 8.71; $\mathrm{N}, 13.38$.

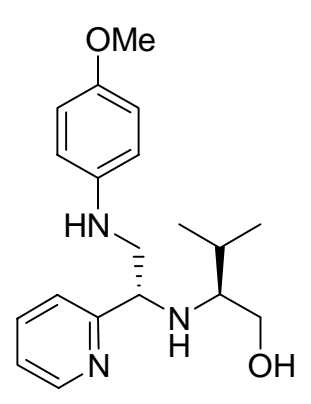

(S)-2-[(S)-2-(4-Methoxyphenylamino)-1-(2-pyridiyl)ethylamino]-3-methyl-1-butanol

(3d):

Yellow oil; $[\alpha]_{\mathrm{D}}{ }^{20}-38.8\left(c\right.$ 1.0, $\left.\mathrm{CHCl}_{3}\right) . \mathrm{IR}$ (neat): $v=3372,2956,2922,2104,1598,1464,1116$. ${ }^{1} \mathrm{H} \mathrm{NMR}\left(300 \mathrm{MHz}, \mathrm{CDCl}_{3}\right) \delta=8.61(\mathrm{ddd}, J=0.9, J=1.8, J=4.8,1 \mathrm{H}), 7.66(\mathrm{dt}, J=1.8, J=7.7$, $1 \mathrm{H}), 7.22(\mathrm{~m}, 2 \mathrm{H}), 6.78(\mathrm{~d}, J=9.0,2 \mathrm{H}), 6.62(\mathrm{~d}, J=9.0,2 \mathrm{H}), 4.02(\mathrm{dd}, J=5.6, J=7.3,1 \mathrm{H})$, $3.75(\mathrm{~s}, 3 \mathrm{H}), 3.61(\mathrm{dd}, J=3.9, J=11.0,1 \mathrm{H}), 3.43(\mathrm{dd}, J=5.4, J=11.0,1 \mathrm{H}), 3.40(\mathrm{dd}, J=5.6, J$ $=12.5,1 \mathrm{H}), 3.29(\mathrm{dd}, J=7.3, J=12.5,1 \mathrm{H}), 2.69(\mathrm{bs}, 3 \mathrm{H}), 2.26(\mathrm{dd}, J=3.9, J=5.4,1 \mathrm{H}), 1.66$ $($ sept, $J=6.8,1 \mathrm{H}), 0.84(\mathrm{~d}, J=6.9,3 \mathrm{H}), 0.80(\mathrm{~d}, J=6.9,3 \mathrm{H}) .{ }^{13} \mathrm{C} \mathrm{NMR}\left(75 \mathrm{MHz}, \mathrm{CDCl}_{3}\right): \delta=$ $161.4,152.3,149.6,142.1,136.5,122.8,122.5,114.9,114.7,63.1,61.1,60.4,55.7,50.9,29.5$ 19.4, 18.9. MS (ES) $m / z=330.2(\mathrm{M}+\mathrm{H})^{+}$. Anal. Calcd for $\mathrm{C}_{19} \mathrm{H}_{27} \mathrm{~N}_{3} \mathrm{O}_{2}: \mathrm{C}, 69.27 ; \mathrm{H}, 8.26 ; \mathrm{N}$, 12.76. Found C, 69.49; H, 8.29; N, 12.73 . 


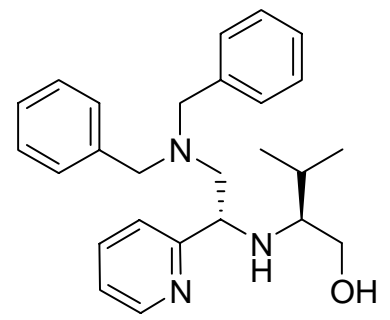

(S)-2-((S)-2-(dibenzylamino)-1-(pyridin-2-yl)ethylamino)-3-methylbutan-1-ol (3e): Yellow oil. $[\alpha]_{\mathrm{D}}{ }^{20}-24.7\left(c 1.7, \mathrm{CHCl}_{3}\right) . \mathrm{IR}(\mathrm{neat}): v=3320,3085,3062,3027,2959,1593,1453,1364,1261$, $1118,1027,780,746,698 .{ }^{1} \mathrm{H}$ NMR $\left(300 \mathrm{MHz}, \mathrm{CDCl}_{3}\right): \delta=8.52(\mathrm{ddd}, J=1.0, J=1.8, J=5.0,1$ H), $7.63(\mathrm{dt}, J=1.9, J=7.7,1 \mathrm{H}), 7.23(\mathrm{td}, J=1.0, J=7.7,1 \mathrm{H}), 7.18(\mathrm{ddd}, J=1.0, J=4.9, J=$ $7.5,1 \mathrm{H}), 3.93(\mathrm{dd}, J=7.2, J=7.2,1 \mathrm{H}), 3.73(\mathrm{~d}, J=13.5,2 \mathrm{H}), 3.66(\mathrm{dd}, J=4.0, J=10.9,1 \mathrm{H})$, $3.58(\mathrm{~d}, J=13.5,2 \mathrm{H}), 3.45(\mathrm{dd}, J=4.9, J=11.0,1 \mathrm{H}), 2.73(\mathrm{~d}, J=7.0,2 \mathrm{H}), 2.18(\mathrm{dd}, J=4.0, J=$ 4.9, $1 \mathrm{H}), 2.09(\mathrm{bs}, 2 \mathrm{H}), 1.61(\mathrm{sept}, J=6.8,1 \mathrm{H}), 0.80(\mathrm{~d}, J=6.8,3 \mathrm{H}), 0.77(\mathrm{~d}, J=6.8,3 \mathrm{H}) .{ }^{13} \mathrm{C}$ NMR $\left(75 \mathrm{MHz}, \mathrm{CDCl}_{3}\right): \delta=162.9,149.1,138.9,136.0,128.0,128.9,128.2,127.0,126.9,122.0$ 62.6, 60.8, 60.7, 60.1, 59.2, 29.7, 19.2, 19.0. MS (ES) $m / z=404.3(\mathrm{M}+\mathrm{H})^{+}$. Anal. Calcd for $\mathrm{C}_{26} \mathrm{H}_{33} \mathrm{~N}_{3} \mathrm{O}: \mathrm{C}, 77.38 ; \mathrm{H}, 8.24 ; \mathrm{N}, 10.41$. Found C, 77.10; H, 8.27; N, 10.37.

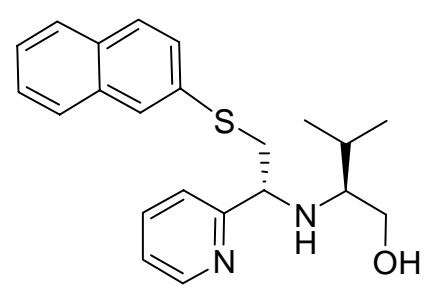

(S)-2-[(R)-2-(2-Naphthylthio)-1-(2-pyridyl)ethylamino]-3-methyl-1-butanol (3f): Yellowish oil; $[\alpha]_{\mathrm{D}}{ }^{20}+30.7\left(c 1.2, \mathrm{CHCl}_{3}\right)$. IR (neat): $v=3421,3342,3052,2951,1581,1463,1434,1043,1014$ 742, 692. ${ }^{1} \mathrm{H}$ NMR $\left(300 \mathrm{MHz}, \mathrm{CDCl}_{3}\right): \delta=8.56(\mathrm{ddd}, J=1.2, J=1.7, J=4.7,1 \mathrm{H}), 7.78(\mathrm{~m}, 2 \mathrm{H})$, $7.74(\mathrm{~m}, 2 \mathrm{H}), 7.62(\mathrm{dt}, J=1.8, J=7.7,1 \mathrm{H}), 7.47(\mathrm{~m}, 2 \mathrm{H}), 7.39(\mathrm{dd}, J=1.8, J=8.7,1 \mathrm{H}), 7.17$ (m, $2 \mathrm{H}), 3.96(\mathrm{dd}, J=6.2, J=6.4,1 \mathrm{H}), 3.61(\mathrm{dd}, J=3.8, J=11.1,1 \mathrm{H}), 3.45(\mathrm{dd}, J=5.0, J=$ $11.1,1 \mathrm{H}), 3.43(\mathrm{dd}, J=6.4, J=13.2,1 \mathrm{H}), 3.37(\mathrm{dd}, J=6.2, J=13.2,1 \mathrm{H}), 2.81(\mathrm{bs}, 2 \mathrm{H}), 2.42$ 
$(\mathrm{ddd}, J=3.8, J=4.8, J=5.0,1 \mathrm{H}), 1.62(\mathrm{sept}, J=6.9,1 \mathrm{H}), 0.76(\mathrm{~d}, J=6.9,3 \mathrm{H}), 0.79(\mathrm{~d}, J=6.9$, $3 \mathrm{H}) .{ }^{13} \mathrm{C} \mathrm{NMR}\left(75 \mathrm{MHz}, \mathrm{CDCl}_{3}\right): \delta=160.1,149.7,136.4,133.7,133.4,131.7,128.5,127.7$, 127.4, 127.1, 127.0, 126.5, 125.7, 122.9, 122.8, 63.3, 61.1, 60.4, 40.4, 29.3, 19.4, 18.7. GC-MS (EI) m/z 262 (100), 230 (55), 263 (51), 115 (46), 78 (27), 104 (15), 264 (12). MS (ES) m/z 367.3 (M + $\mathrm{H})^{+}, 389.0(\mathrm{M}+\mathrm{Na})^{+}$. Anal. Calcd for $\mathrm{C}_{22} \mathrm{H}_{26} \mathrm{~N}_{2} \mathrm{OS}: \mathrm{C}, 72.09 ; \mathrm{H}, 7.15 ; \mathrm{N}, 7.64$. Found C, 72.15; H, 7.17; N, 7.61.

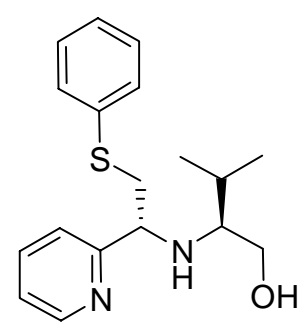

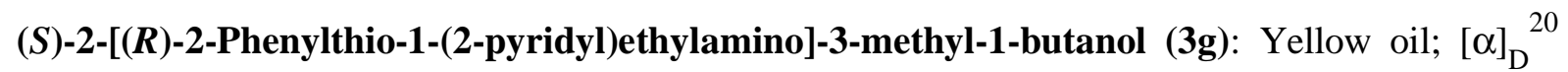
$+11.4\left(c\right.$ 0.9, $\left.\mathrm{CHCl}_{3}\right)$. IR (neat): $v=3424,3345,3057,2959,2925,2972,1643,1589,1467,1436$, 1258, 1042, 1013, 743, 690. ${ }^{1} \mathrm{H}$ NMR $\left(300 \mathrm{MHz}, \mathrm{CDCl}_{3}\right): \delta=8.61(\mathrm{dt}, J=1.6, J=4.4,1 \mathrm{H}), 7.66$ $(\mathrm{dt}, J=1.8, J=7.8,1 \mathrm{H}), 7.38(\mathrm{~m}, 1 \mathrm{H}), 7.31(\mathrm{~m}, 2 \mathrm{H}), 7.21(\mathrm{~m}, 2 \mathrm{H}), 3.92(\mathrm{dd}, J=6.3, J=7.2,1$ H), $3.62(\mathrm{dd}, J=3.8, J=11.1,1 \mathrm{H}), 3.46(\mathrm{dd}, J=5.0, J=11.1,1 \mathrm{H}), 3.35(\mathrm{dd}, J=7.2, J=13.2,1$ H), $3.29(\mathrm{dd}, J=6.3, J=13.2,1 \mathrm{H}), 2.55(\mathrm{bs}, 2 \mathrm{H}), 2.21(\mathrm{ddd}, J=3.8, J=5.0, J=6.4,1 \mathrm{H}), 1.63$ $(\mathrm{sept}, J=6.9,1 \mathrm{H}), 0.82(\mathrm{~d}, J=6.9,3 \mathrm{H}), 0.78(\mathrm{~d}, J=6.9,6 \mathrm{H}) .{ }^{13} \mathrm{C} \mathrm{NMR}\left(75 \mathrm{MHz}, \mathrm{CDCl}_{3}\right): \delta=$ $161.3,149.7,136.3,129.4,128.9,126.1,122.7,122.6,63.0,61.1,60.6,41.1,29.4,19.4,18.8 . \mathrm{GC}-$ MS m/z 193 (100), 107 (39), 214 (35), 106 (17), 175 (12), 92 (12), 119 (11), 78 (10), 136 (9), 285 (3). Anal. Calcd for $\mathrm{C}_{17} \mathrm{H}_{23} \mathrm{~N}_{2} \mathrm{OS}$ : C, 67.29; H, 7.64; N, 9.23. Found C, 67.58; H, 7.67; N, 9.22. 


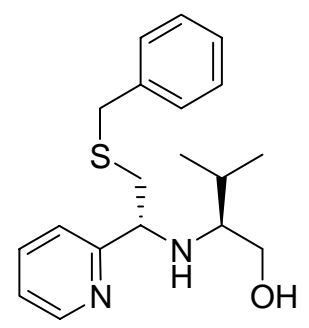

(S)-2-[(R)-2-Benzylthio-1-(2-pyridyl)ethylamino]-3-methyl-1-butanol (3h): Yellow oil; $[\alpha]_{\mathrm{D}}^{20}$ $-23.4\left(c 1.2, \mathrm{CHCl}_{3}\right)$. IR (neat): $v=3382,3060,3030,2951,2922,1590,1491,1472,1044,741$, 702. ${ }^{1} \mathrm{H}$ NMR $\left(300 \mathrm{MHz}, \mathrm{CDCl}_{3}\right): \delta=8.57(\mathrm{ddd}, J=0.9, J=1.7, J=4.9,1 \mathrm{H}), 7.63(\mathrm{dt}, J=1.7, J$ $=7.6,1 \mathrm{H}), 7.32(\mathrm{~m}, 5 \mathrm{H}), 7.26(\mathrm{ddd}, J=0.9, J=4.9, J=7.6,1 \mathrm{H}), 7.13 \mathrm{~d}, J=7.6,1 \mathrm{H}), 3.77(\mathrm{dd}, J$ $=6.3, J=6.3,1 \mathrm{H}), 3.68(\mathrm{~s}, 2 \mathrm{H}), 3.61(\mathrm{dd}, J=3.7, J=11.1,1 \mathrm{H}), 3.46(\mathrm{dd}, J=5.1, J=11.1,1 \mathrm{H})$, $2.86(\mathrm{dd}, J=6.3, J=13.3,1 \mathrm{H}), 2.77(\mathrm{dd}, J=6.3, J=13.3,1 \mathrm{H}), 2.36(\mathrm{bs}, 2 \mathrm{H}), 2.39(\mathrm{dd}, J=3.7, J$ $=5.1,1 \mathrm{H}), 1.62(\mathrm{sept}, J=7.0,1 \mathrm{H}), 0.80(\mathrm{~d}, J=7.0,3 \mathrm{H}), 0.77(\mathrm{~d}, J=7.0,6 \mathrm{H}) .{ }^{13} \mathrm{C}$ NMR $(75$ $\left.\mathrm{MHz}, \mathrm{CDCl}_{3}\right): \delta=159.7,149.6,138.3,136.3,128.9,128.5,127.0,122.4,122.5,63.0,61.4,60.6$ 38.8, 37.1, 29.4, 19.4, 18.8. GC-MS m/z 91 (100), 193 (92), 107 (31), 106 (31), 228 (16), 124 (14), 65 (14), 281 (12), 136 (12), 78 (12), 175 (11), 51 (10), 299 (4), 207 (3). Anal. Calcd for $\mathrm{C}_{19} \mathrm{H}_{26} \mathrm{~N}_{2} \mathrm{OS}: \mathrm{C}, 69.05 ; \mathrm{H}, 7.93 ; \mathrm{N}, 8.48$. Found C, 69.26; H, 7.96; N, 8.45.

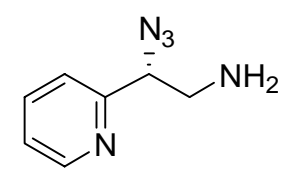

(R)-2-azido-2-(2-pyridyl)ethanamine: Red oil; IR (neat): $v=3365,2962,2923,2102,1590,1571$, 1472, 1436, 1260, 1094, 1020, 798. ${ }^{1} \mathrm{H}$ NMR $\left(300 \mathrm{MHz}, \mathrm{CDCl}_{3}\right): \delta=8.60(\mathrm{~d}, J=4.7,1 \mathrm{H}), 7.72$ $(\mathrm{dt}, J=1.6, J=7.9,1 \mathrm{H}), 7.35(\mathrm{~d}, J=7.9,1 \mathrm{H}), 7.24(\mathrm{ddd}, J=1.6, J=4.7, J=7.9,1 \mathrm{H}), 4.55(\mathrm{dd}, J$ $=5.5, J=13.3,1 \mathrm{H}), 3.18(\mathrm{dd}, J=5.5, J=13.3,1 \mathrm{H}), 3.06(\mathrm{dd}, J=7.1, J=13.3,1 \mathrm{H}), 1.76(\mathrm{bs}, 2$ H). ${ }^{13} \mathrm{C}$ NMR $\left(75 \mathrm{MHz}, \mathrm{CDCl}_{3}\right): \delta=157.4,149.6,136.9,123.0,121.7,69.0,46.3 . \mathrm{MS}(\mathrm{ES}) \mathrm{m} / \mathrm{z}$ $164.3(\mathrm{M}+\mathrm{H})^{+}$ 


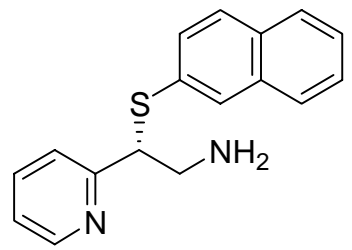

(R)-2-(2-Naphthylthio)-2-(2-pyridyl)ethanamine: Yellowish oil; ${ }^{1} \mathrm{H} \quad \mathrm{NMR} \quad(300 \mathrm{MHz}$, $\left.\mathrm{CDCl}_{3}\right): \delta=8.56(\mathrm{ddd}, J=0.9, J=1.8, J=4.9,1 \mathrm{H}), 7.79(\mathrm{~m}, 2 \mathrm{H}), 7.71(\mathrm{~m}, 2 \mathrm{H}), 7.61(\mathrm{td}, J=1.9$, $J=7.8,1 \mathrm{H}), 7.44(\mathrm{~m}, 4 \mathrm{H}), 7.32(\mathrm{dt}, J=0.9, J=7.7,1 \mathrm{H}), 7.14(\mathrm{ddd}, J=1.1, J=4.8, J=7.8,1 \mathrm{H})$, $4.49(\mathrm{dd}, J=6.1, J=7.2,1 \mathrm{H}), 3.39(\mathrm{dd}, J=7.2, J=13.5,1 \mathrm{H}), 3.27(\mathrm{dd}, J=6.1, J=13.5,1 \mathrm{H})$. ${ }^{13} \mathrm{C}$ NMR $\left(75 \mathrm{MHz}, \mathrm{CDCl}_{3}\right): \delta=159.5,149.4,136.5,134.1,130.5,128.8,127.5,127.3,126.9$, 126.6, 126.3, 126.0, 125.5, 122.9, 122.2, 57.7, 46.0. GC-MS (EI) m/z 262 (100), 230 (49), 263 (44), 115 (42), 78 (31), 51 (14), 217 (11), 160 (8).

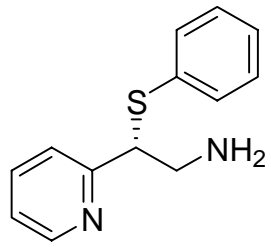

(R)-2-(2-Phenylthio)-2-(2-pyridyl)ethanamine: Yellow oil; ${ }^{1} \mathrm{H}$ NMR $\left(300 \mathrm{MHz}, \mathrm{CDCl}_{3}\right): \delta=8.56$ $(\mathrm{ddd}, J=1.0, J=1.8, J=4.8,1 \mathrm{H}), 7.61(\mathrm{dt}, J=1.8, J=7.7,1 \mathrm{H}), 7.33(\mathrm{~m}, 2 \mathrm{H}), 7.29(\mathrm{~m}, 2 \mathrm{H})$, $7.19(\mathrm{~m}, 2 \mathrm{H}), 7.16(\mathrm{~m}, 1 \mathrm{H}), 4.33(\mathrm{dd}, J=6.1, J=7.2,1 \mathrm{H}), 3.31(\mathrm{dd}, J=7.2, J=13.4,1 \mathrm{H}), 3.19$ $(\mathrm{dd}, J=6.1, J=13.4,1 \mathrm{H}) .{ }^{13} \mathrm{C} \mathrm{NMR}\left(75 \mathrm{MHz}, \mathrm{CDCl}_{3}\right): \delta=170.8,159.6,149.4,136.5,134.1$, 131.9, 128.8, 128.6, 124.6, 122.9, 122.2, 57.8, 46.0. GC-MS (EI) m/z 168 (100), 201 (99), 124 (20), 65 (17), 78 (16), 94 (15), $212(9), 109$ (9), 186 (4). 


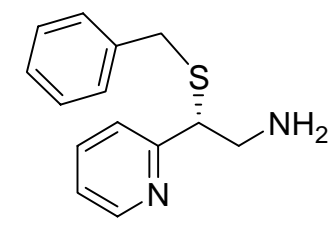

(R)-2-(Benzylthio)-2-(2-pyridyl)ethanamine: Yellow oil; ${ }^{13} \mathrm{H}$ NMR $\left(300 \mathrm{MHz}, \mathrm{CDCl}_{3}\right): \delta=8.60$ $(\mathrm{d}, J=4.4,1 \mathrm{H}), 7.68(\mathrm{dt}, J=1.8, J=7.6,1 \mathrm{H}), 7.30(\mathrm{~m}, 7 \mathrm{H}), 3.90(\mathrm{dd}, J=6.3, J=7.0,1 \mathrm{H}), 3.72$ $(\mathrm{d}, J=13.2,1 \mathrm{H}), 3.64(\mathrm{~d}, J=13.2,1 \mathrm{H}), 3.25(\mathrm{dd}, J=7.0, J=13.4,1 \mathrm{H}), 3.16(\mathrm{dd}, J=6.3, J=$ 13.4, $1 \mathrm{H}), 1.94$ (bs, $2 \mathrm{H}) .{ }^{13} \mathrm{C} \mathrm{NMR}\left(75 \mathrm{MHz}, \mathrm{CDCl}_{3}\right): \delta=160.4,149.3,136.7,130.3,128.9$, 128.5, 127.0, 122.9, 122.1, 54.0, 45.8, 35.3. GC-MS m/z 124 (100), 91 (28), 215 (16), $122(14), 105$ (11), 106 (10), 65 (10), 79 (9), 227 (2).

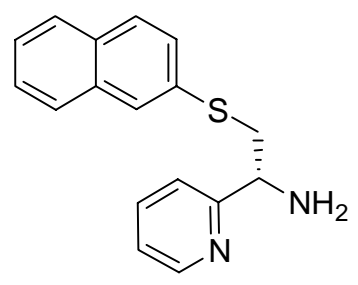

(R)-2-(2-Naphthylthio)-1-(2-pyridyl)ethanamine: Yellowish oil; ${ }^{1} \mathrm{H} \quad \mathrm{NMR} \quad(300 \mathrm{MHz}$, $\left.\mathrm{CDCl}_{3}\right): \delta=8.59(\mathrm{~m}, 1 \mathrm{H}), 7.80(\mathrm{~m}, 2 \mathrm{H}), 7.76(\mathrm{dt}, J=1.8, J=7.7,1 \mathrm{H}), 7.49(\mathrm{~m}, 3 \mathrm{H}), 7.33(\mathrm{~m}, 2$ H), $7.18(\mathrm{ddd}, J=0.7, J=4.8, J=7.7,1 \mathrm{H}), 4.23(\mathrm{dd}, J=5.4, J=8.2,1 \mathrm{H}), 3.57(\mathrm{dd}, J=5.4, J=$ 13.4, $1 \mathrm{H}), 3.32(\mathrm{dd}, J=8.2, J=13.3,1 \mathrm{H}), 2.44(\mathrm{bs}, 2 \mathrm{H}) .{ }^{13} \mathrm{C} \mathrm{NMR}\left(75 \mathrm{MHz}, \mathrm{CDCl}_{3}\right): \delta=162.5$, $149.7,136.9,133.9,129.2,128.8,127.9,127.8,127.7,127.4,126.8,126.7,126.1,122.8,121.9$ $56.2,42.7$. 


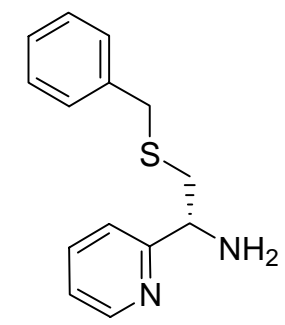

(R)-2-Benzylthio-1-(2-pyridyl)ethanamine: Yellow oil; ${ }^{1} \mathrm{H}$ NMR $\left(300 \mathrm{MHz}, \mathrm{CDCl}_{3}\right): \delta=8.57$ (m, $1 \mathrm{H}), 7.67(\mathrm{~m}, 1 \mathrm{H}), 7.31(\mathrm{~m}, 6 \mathrm{H}), 7.21(\mathrm{~m}, 1 \mathrm{H}), 4.56(\mathrm{dd}, J=6.8, J=7.8,1 \mathrm{H}), 3.67(\mathrm{~s}, 2 \mathrm{H})$, $3.12(\mathrm{dd}, J=6.8, J=12.9,1 \mathrm{H}), 2.75(\mathrm{dd}, J=6.8, J=12.9,1 \mathrm{H}), 2.14(\mathrm{bs}, 2 \mathrm{H}) .{ }^{13} \mathrm{C}$ NMR $(75$ $\left.\mathrm{MHz}, \mathrm{CDCl}_{3}\right): \delta=162.4,149.4,136.5,135.7,129.7,129.4,129.0,126.2,122.4,121.6,55.9,42.7$. GC-MS (EI) m/z 107 (100), 80 (20), 213 (4), 136 (4), 230 (2), 183 (2).

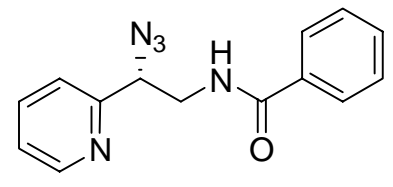

(R)-N-[2-Azido-2-(2-pyridyl)ethyl]benzamide (6b): Red oil; $[\alpha]_{\mathrm{D}}{ }^{20}+55.5\left(c \quad 1.1, \mathrm{CHCl}_{3}\right)$. IR (neat): $v=3288,3068,2937,2104,1638,1540,1321,772,707 .{ }^{1} \mathrm{H}$ NMR $\left(300 \mathrm{MHz}, \mathrm{CDCl}_{3}\right): \delta=$ $8.64(\mathrm{dt}, J=0.7, J=4.8,1 \mathrm{H}), 8.11(\mathrm{~d}, J=6.9,1 \mathrm{H}), 7.77(\mathrm{~m}, 2 \mathrm{H}), 7.48(\mathrm{~m}, 1 \mathrm{H}), 7.42(\mathrm{~m}, 3 \mathrm{H})$, $7.31(\mathrm{ddt}, J=0.8, J=4.9, J=7.6,1 \mathrm{H}), 7.16(\mathrm{~m}, 1 \mathrm{H}), 4.90(\mathrm{t}, J=6.1,1 \mathrm{H}), 4.07(\mathrm{ddd}, J=6.1, J=$ $6.9, J=12.4,1 \mathrm{H}), 3.87(\mathrm{ddd}, J=6.1, J=6.9, J=12.4,1 \mathrm{H}) .{ }^{13} \mathrm{C} \mathrm{NMR}\left(75 \mathrm{MHz}, \mathrm{CDCl}_{3}\right): \delta=$ $167.7,149.4,137.5,134.1,133.2,131.6,129.9,128.5,128.3,126.9,123.6,122.6,64.1,43.3$. GC$\operatorname{MS}\left(280{ }^{\circ} \mathrm{C}\right) \mathrm{m} / z 105$ (100), 218 (67), 77 (61), 262 (56), 263 (29), 115 (26), 219 (24), 230 (23), 384 (13), 160 (12). MS (ES) $m / z 268.2(\mathrm{M}+\mathrm{H})^{+}, 290.1(\mathrm{M}+\mathrm{Na})^{+}, 557.2(2 \mathrm{M}+\mathrm{Na})^{+}$. Anal. Calcd for $\mathrm{C}_{14} \mathrm{H}_{13} \mathrm{~N}_{5} \mathrm{O}: \mathrm{C}, 62.91 ; \mathrm{H}, 4.90 ; \mathrm{N}, 26.20$. Found C, 63.10; H, 4.92; N, 26.12. 


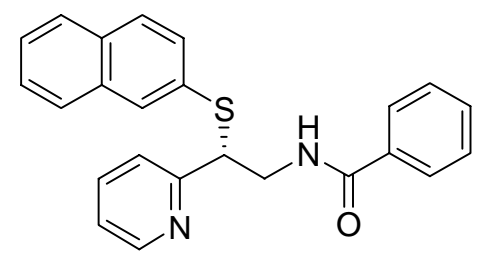

(R)-N-[2-(2-Naphthylthio)-2-(2-pyridyl)ethyl]benzamide (6f): White crystals. (from $\left.\mathrm{Et}_{2} \mathrm{O}\right)$; m.p. $=122.0-122.5^{\circ} \mathrm{C} ;[\alpha]_{\mathrm{D}}{ }^{20}+58.7\left(c 1.9, \mathrm{CHCl}_{3}\right) . \mathrm{IR}(\mathrm{KBr}): v=3326,3046,3003,2926,1843,1710$, $1638,1578,1523,1436,1271,822,745,701 .{ }^{1} \mathrm{H}$ NMR $\left(300 \mathrm{MHz}, \mathrm{CDCl}_{3}\right): \delta=8.56(\mathrm{ddd}, J=0.7$ $J=1.6, J=4.9,1 \mathrm{H}), 7.93(\mathrm{~s}, 1 \mathrm{H}), 7.73(\mathrm{~m}, 5 \mathrm{H}), 7.58(\mathrm{dt}, J=1.9, J=7.8,1 \mathrm{H}), 7.45(\mathrm{~m}, 5 \mathrm{H})$, $7.32(\mathrm{~m}, 3 \mathrm{H}), 7.16(\mathrm{ddd}, J=0.7, J=4.9, J=7.8,1 \mathrm{H}), 4.85(\mathrm{t}, J=6.6,1 \mathrm{H}), 4.21(\mathrm{ddd}, J=6.1, J=$ $6.6, J=13.5,1 \mathrm{H}), 4.15(\mathrm{ddd}, J=6.1, J=6.6, J=13.5,1 \mathrm{H}) .{ }^{13} \mathrm{C} \mathrm{NMR}\left(75 \mathrm{MHz}, \mathrm{CDCl}_{3}\right): \delta=$ $167.4,158.9,149.0,136.9,133.5,132.2,131.2,131.0,129.2,128.5,128.3,127.5,127.4,126.8$ 126.4, 126.2, 123.6, 122.5, 52.3, 43.2. GC-MS (EI) $\left(280{ }^{\circ} \mathrm{C}\right) \mathrm{m} / z 384$ (4), 263 (30), 230 (29), 211 (18), 160 (8), $136(16), 115(13), 105$ (100), $77(38) . \mathrm{MS}(\mathrm{ES}) \mathrm{m} / z=385.1(\mathrm{M}+\mathrm{H})^{+}, 791.0(2 \mathrm{M}+$ $\mathrm{Na})^{+}$. Anal. Calcd for $\mathrm{C}_{24} \mathrm{H}_{20} \mathrm{~N}_{2} \mathrm{OS}: \mathrm{C}, 74.97 ; \mathrm{H}, 5.24 ; \mathrm{N}, 7.29$. Found C, 75.05; H, 5.26; N, 7.28.

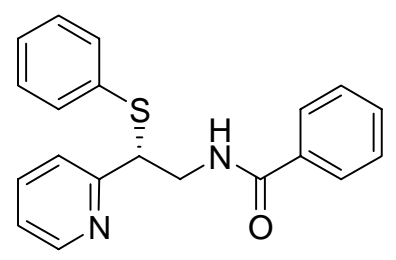

(R)-N-[2-Phenylthio-2-(2-pyridyl)ethyl]benzamide (6g): $\operatorname{Red~oil;~}[\alpha]_{\mathrm{D}}{ }^{20}+51.8\left(c 2.0, \mathrm{CHCl}_{3}\right)$. IR (neat): $v=3323,3058,2925,2853,1644,1579,1537,1484,1436,1292,1075,749,692 .{ }^{1} \mathrm{H}$ NMR $\left(300 \mathrm{MHz}, \mathrm{CDCl}_{3}\right): \delta=8.61(\mathrm{~d}, J=4.6,1 \mathrm{H}), 7.74(\mathrm{~m}, 2 \mathrm{H}), 7.68(\mathrm{td}, J=1.7, J=7.7,1 \mathrm{H}), 7.49$ (m, $2 \mathrm{H}), 7.44(\mathrm{~m}, 3 \mathrm{H}), 7.32(\mathrm{~m}, 2 \mathrm{H}), 7.24(\mathrm{~m}, 2 \mathrm{H}), 4.72(\mathrm{dd}, J=6.1, J=6.9,1 \mathrm{H}), 4.16(\mathrm{~m}, 2 \mathrm{H})$. ${ }^{13} \mathrm{C} \mathrm{NMR}\left(75 \mathrm{MHz}, \mathrm{CDCl}_{3}\right): \delta=167.4,159.0,149.1,137.0,134.4,133.5,132.5,131.4,129.0$, 128.5, 127.7, 126.9, 123.8, 122.4, 52.5, 43.0. GC-MS (EI) m/z 105 (100), 77 (73), 168 (61), 213 
(38), 201 (49), 334 (20), 180 (16), 225 (14), 119 (8). Anal. Calcd for $\mathrm{C}_{20} \mathrm{H}_{18} \mathrm{~N}_{2} \mathrm{OS}$ : C, 71.83; H, 5.42; N, 8.38. Found C, 71.58; H, 5.43; N, 8.35.

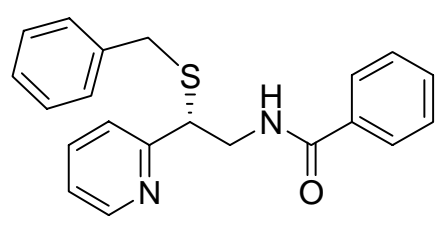

(R)- $N$-[2-Benzylthio-2-(2-pyridyl)ethyl]benzamide $(6 \mathbf{6 h})$ : Colourless oil; $[\alpha]_{\mathrm{D}}{ }^{20}+26.1(c \quad 1.0$, $\left.\mathrm{CHCl}_{3}\right) . \mathrm{IR}$ (neat): $v=3320,3060,2923,1644,1537,1471,1434,1290,697 .{ }^{1} \mathrm{H} \mathrm{NMR}(300 \mathrm{MHz}$, $\left.\mathrm{CDCl}_{3}\right): \delta=8.59(\mathrm{ddd}, J=1.0, J=1.9, J=4.9,1 \mathrm{H}), 7.76(\mathrm{~m}, 2 \mathrm{H}), 7.69(\mathrm{dt}, J=1.9, J=7.8,1 \mathrm{H})$, $7.47(\mathrm{~m}, 5 \mathrm{H}), 7.32(\mathrm{~m}, 4 \mathrm{H}), 7.25(\mathrm{~m}, 1 \mathrm{H}), 4.23(\mathrm{dd}, J=6.7, J=9.7,1 \mathrm{H}), 4.17(\mathrm{ddd}, J=4.6, J=$ $6.7, J=13.1,1 \mathrm{H}), 3.97(\mathrm{ddd}, J=4.6, J=9.7, J=13.1,1 \mathrm{H}), 3.89(\mathrm{~d}, J=13.5,1 \mathrm{H}), 3.75(\mathrm{~d}, J=$ 13.5, $1 \mathrm{H}) .{ }^{13} \mathrm{C} \mathrm{NMR}\left(75 \mathrm{MHz}, \mathrm{CDCl}_{3}\right): \delta=167.4,159.7,148.6,137.9,137.3,134.5,131.4,129.0$, 128.6, 128.5, 127.1, 127.0, 123.8, 122.5, 48.7, 42.4, 35.7. GC-MS (EI) m/z 105 (100), 124 (76), 226 (74), 77 (49), 91 (45), 257 (41). Anal. Calcd for $\mathrm{C}_{21} \mathrm{H}_{20} \mathrm{~N}_{2} \mathrm{OS}$ : C, 72.38; H, 5.79; N, 8.04. Found C, 72.40; H, 5.80; N, 8.02.

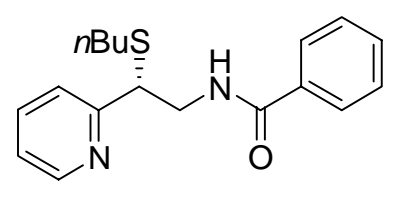

(R)- $N$-[(2-Butylthio-2-(2-pyridyl)ethyl]benzamide $(6 \mathbf{6})$ : Yellow oil; $[\alpha]_{\mathrm{D}}{ }^{20}+27.4\left(\right.$ c $\left.1.0, \mathrm{CHCl}_{3}\right)$; $3314,3057,2913,1647,1534,1477,1297,694 .{ }^{1} \mathrm{H}$ NMR $\left(300 \mathrm{MHz}, \mathrm{CDCl}_{3}\right): \delta=8.58(\mathrm{ddd}, J=$ $0.9, J=1.7, J=4.9,1 \mathrm{H}), 7.74(\mathrm{~m}, 2 \mathrm{H}), 7.71(\mathrm{dt}, J=1.7, J=7.4,1 \mathrm{H}), 7.47(\mathrm{~m}, 1 \mathrm{H}), 7.42(\mathrm{~m}, 2$ H), $7.23(\mathrm{ddd}, J=0.9, J=4.7, J=7.5,1 \mathrm{H}), 4.29(\mathrm{dd}, J=5.8, J=7.4,1 \mathrm{H}), 4.11(\mathrm{ddd}, J=6.2, J=$ 7.4, $J=13.6,1 \mathrm{H}), 4.01(\mathrm{ddd}, J=5.8, J=11.2, J=13.6,1 \mathrm{H}), 2.57(\mathrm{~m}, 2 \mathrm{H}), 1,54(\mathrm{~m}, 2 \mathrm{H}), 1,38$ $(\mathrm{m}, 2 \mathrm{H}), 0.87(\mathrm{t}, J=7.3,3 \mathrm{H}) .{ }^{13} \mathrm{C} \mathrm{NMR}\left(75 \mathrm{MHz}, \mathrm{CDCl}_{3}\right): \delta=167.4,160.0,148.7,137.4,134.4$, 
$133.3,131.4,130.1,128.5,128.4,126.9,123.7,122.5,49.2,42.8,31.6,30.9,21.9,13.6 . \mathrm{MS}(\mathrm{EI})$ m/z 314 (4), 257 (16), 181 (11), 160 (18), 124 (57), 105 (100), 93 (14), 77 (55). MS (ES) m/z = $315.2(\mathrm{M}+\mathrm{H})^{+}, 651.3(2 \mathrm{M}+\mathrm{Na})^{+}$. Anal. Calcd for $\mathrm{C}_{18} \mathrm{H}_{22} \mathrm{~N}_{2} \mathrm{OS}$ : C, 68.75; $\mathrm{H}, 7.05 ; \mathrm{N}, 8.91$. Found C, 68.38; H, 7.08; N, 8.89.

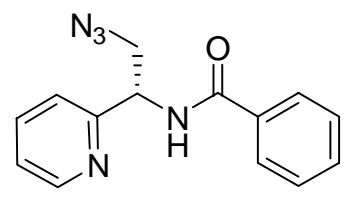

$N$-((S)-2-azido-1-(pyridin-2-yl)ethyl)benzamide (7b): Yellow oil; $[\alpha]_{\mathrm{D}}{ }^{20}+13.4\left(c\right.$ 1.2, $\left.\mathrm{CHCl}_{3}\right)$; IR (neat): $v=3282,3071,2932,2108,1631,1547,1315,778 .{ }^{1} \mathrm{H}$ NMR $\left(300 \mathrm{MHz}, \mathrm{CDCl}_{3}\right): \delta=8.63$ $(\mathrm{ddd}, J=0.8, J=1.6, J=4.8,1 \mathrm{H}), 7.88(\mathrm{~m}, 2 \mathrm{H}), 7.75(\mathrm{dd}, J=1.9, J=7.7,1 \mathrm{H}), 7.45(\mathrm{~m}, 3 \mathrm{H})$, $7.38(\mathrm{dt}, J=0.8, J=1.6,1 \mathrm{H}), 7.30(\mathrm{ddd}, J=1.9, J=4.8, J=7.4,1 \mathrm{H}), 5.46(\mathrm{ddd}, J=4.9, J=5.7, J$ $=7.3,1 \mathrm{H}), 3.85(\mathrm{dd}, J=4.9, J=12.2,1 \mathrm{H}), 3.80(\mathrm{dd}, J=5.7, J=12.2,1 \mathrm{H}) .{ }^{13} \mathrm{C} \mathrm{NMR}(75 \mathrm{MHz}$, $\left.\mathrm{CDCl}_{3}\right): \delta=167.0,156.4,149.3,13.1,134.0,131.8,128.6,127.1,127.0,123.2,122.6,55.0,53.5$. MS (ES) $m / z=268.2(\mathrm{M}+\mathrm{H})^{+}$. Anal. Calcd for $\mathrm{C}_{14} \mathrm{H}_{13} \mathrm{~N}_{5} \mathrm{O}: \mathrm{C}, 62.91 ; \mathrm{H}, 4.90 ; \mathrm{N}, 26.20$. Found $\mathrm{C}$, 63.21; H, 4.92; N, 26.18.

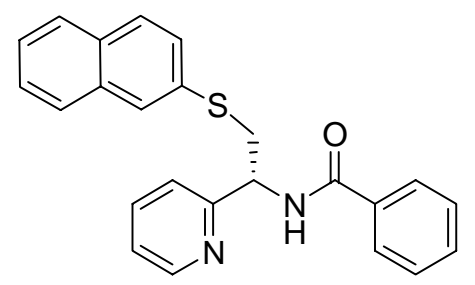

(R)- $N$-[2-Naphthylthio)-1-(2-pyridyl)ethyl]benzamide (7f): White crystals (from $\mathrm{Et}_{2} \mathrm{O}$ ); m.p. = $119.5-120^{\circ} \mathrm{C} .[\alpha]_{\mathrm{D}}{ }^{20}-22.7\left(c 1.3, \mathrm{CHCl}_{3}\right)$. IR (Nujol): $v=3310,1638,1518,1468,1370,810,729$. ${ }^{1} \mathrm{H}$ NMR $\left(300 \mathrm{MHz}, \mathrm{CDCl}_{3}\right): \delta=8.58(\mathrm{ddd}, J=0.8, J=1.6, J=4.8,1 \mathrm{H}), 8.12(\mathrm{~d}, J=7.0,1 \mathrm{H})$; 7.81 (m, 2 H), 7.75 (m, 3 H), 7.63 (m, 2 H), 7.47 (m, 3 H), 7.38 (m, 2 H), 7.20 (m, 2 H), 5.56 (dd, $J$ $=5.2, J=6.8,1 \mathrm{H}), 3.82(\mathrm{dd}, J=5.2, J=13.6,1 \mathrm{H}), 3.51(\mathrm{dd}, J=7.8, J=13.6,1 \mathrm{H}) .{ }^{13} \mathrm{C} \mathrm{NMR}(75$ 
$\left.\mathrm{MHz}, \mathrm{CDCl}_{3}\right): \delta=166.8,157.6,149.0,136.9,133.7,133.5,131.5,130.1,128.5,128.4,127.6$, $127.2,127.1,126.5,125.7,123.4,123.6,123.1,53.9,38.5 . \mathrm{MS}(\mathrm{ES}) \mathrm{m} / z 385.1(\mathrm{M}+\mathrm{H})^{+}, 791.0(2$ $\mathrm{M}+\mathrm{Na})^{+}$. Anal. Calcd for $\mathrm{C}_{24} \mathrm{H}_{20} \mathrm{~N}_{2} \mathrm{OS}: \mathrm{C}, 74.97 ; \mathrm{H}, 5.24 ; \mathrm{N}, 7.29$. Found $\mathrm{C}, 75.10 ; \mathrm{H}, 5.26 ; \mathrm{N}$, 7.27 .

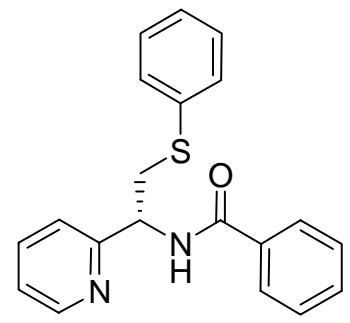

(R)- $N$-[2-Phenylthio-1-(pyridyl)ethyl]benzamide (7g): Red oil; $[\alpha]_{\mathrm{D}}{ }^{20}-19.2\left(c 0.4, \mathrm{CHCl}_{3}\right)$. IR (neat): $v=3321,3054,2927,2852,1642,1572,1533,1482,1431,1290,1078,747,691 .{ }^{1} \mathrm{H}$ NMR $\left(300 \mathrm{MHz}, \mathrm{CDCl}_{3}\right): \delta=8.58(\mathrm{ddd}, J=0.9, J=1.7, J=4.9,1 \mathrm{H}), 7.82(\mathrm{~m}, 2 \mathrm{H}), 7.68(\mathrm{dt}, J=1.8, J$ $=7.8,1 \mathrm{H}), 7.49(\mathrm{~m}, 3 \mathrm{H}), 7.42(\mathrm{~m}, 3 \mathrm{H}), 7.33(\mathrm{dt}, J=0.9, J=7.8,1 \mathrm{H}), 7.27(\mathrm{~m}, 2 \mathrm{H}), 7.15(\mathrm{dt}, J=$ $1.2, J=6.5,1 \mathrm{H}), 5.48(\mathrm{dd}, J=5.1, J=7.2, J=8.0,1 \mathrm{H}), 3.72(\mathrm{dd}, J=5.1, J=13.6,1 \mathrm{H}), 3.44(\mathrm{dd}$, $J=8.0, J=13.6,1 \mathrm{H}) .{ }^{13} \mathrm{C} \mathrm{NMR}\left(75 \mathrm{MHz}, \mathrm{CDCl}_{3}\right): \delta=166.7,157.5,137.1,134.1,131.6,129.2$, 129.0, 128.5, 127.1, 126.1, 123.6, 123.1, 53.6, 38.7. GC-MS (EI) m/z 105 (100), 77 (69), 213 (68), 136 (47), 211 (31), 212 (26), 180 (11), 281 (5), 334 (4). Anal. Calcd for $\mathrm{C}_{20} 18_{3} \mathrm{~N}_{2} \mathrm{OS}: \mathrm{C}, 71.83 ; \mathrm{H}$, 5.42; N, 8.38. Found C, 71.98; H, 5.41; N, 8.36.

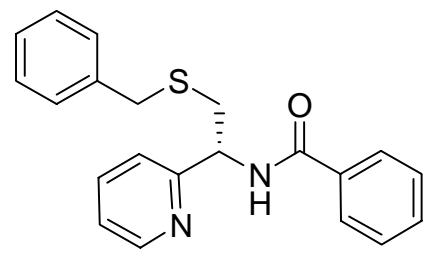

(R)- $N$-[2-(Benzylthio)-1-(2-pyridyl)ethyl]benzamide (7h): Colourless oil; $[\alpha]_{D}{ }^{20}-17.1(c 0.5$, $\left.\mathrm{CHCl}_{3}\right) . \mathrm{IR}$ (neat): $v=3320,3060,2923,1645,1589,1536,1434,1290,696 .{ }^{1} \mathrm{H}$ NMR $(300 \mathrm{MHz}$, 
$\left.\mathrm{CDCl}_{3}\right): \delta=8.60(\mathrm{ddd}, J=0.8, J=1.7, J=4.9,1 \mathrm{H}), 7.89(\mathrm{~m}, 2 \mathrm{H}), 7.48(\mathrm{~m}, 2 \mathrm{H}), 7.39(\mathrm{~m}, 2 \mathrm{H})$, $7.29(\mathrm{~m}, 7 \mathrm{H}), 5.46(\mathrm{dd}, J=5.9, J=7.5,1 \mathrm{H}), 3.65(\mathrm{~s}, 2 \mathrm{H}), 3.14(\mathrm{dd}, J=5.7, J=13.5,1 \mathrm{H}) 3.04$ $(\mathrm{ddd}, J=7.5, J=13.6,1 \mathrm{H}) .{ }^{13} \mathrm{C} \mathrm{NMR}\left(75 \mathrm{MHz} \mathrm{CDCl}_{3}\right): \delta=168.5,158.9,147.8,137.7,137.2$, 134.7, 131.7, 129.0, 128.6, 127.3, 127.2, 123.6, 122.4, 53.1, 36.7, 29.7. GC-MS (EI) m/z 105 (100), 257 (52), 77 (36), 91 (20), 136 (18), 211 (10), 258 (8). Anal. Calcd for $\mathrm{C}_{21} \mathrm{H}_{20} \mathrm{~N}_{2} \mathrm{OS}$ : C, 72.38 ; $\mathrm{H}$, 5.79; N, 8.04. Found C, 72.11; H, 5.80; N, 8.03.

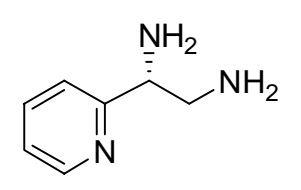

(R)-1-(2-Pyridyl)ethane-1,2-diamine (8): Yellow oil; $[\alpha]_{\mathrm{D}}{ }^{20}-45.6\left(c 0.7, \mathrm{CHCl}_{3}\right)$. IR (neat): $v=$ 3361, 2968, 2922, 2101, 1476, 1435, 1092, 1016, 794. ${ }^{1} \mathrm{H}$ NMR $\left(300 \mathrm{MHz}, \mathrm{CDCl}_{3}\right): \delta=8.54(\mathrm{ddd}$, $J=0.7, J=1.7, J=7.9,1 \mathrm{H}), 7.69(\mathrm{dt}, J=1.7, J=7.8,1 \mathrm{H}), 7.32(\mathrm{~d}, J=7.9,1 \mathrm{H}), 7.21(\mathrm{ddd}, J=$ $0.9, J=4.7, J=7.9,1 \mathrm{H}), 3.99(\mathrm{dd}, J=5.2, J=7.0,1 \mathrm{H}), 3.08(\mathrm{dd}, J=5.2, J=12.7,1 \mathrm{H}), 2.93(\mathrm{dd}$, $J=7.0, J=12.7,1 \mathrm{H}), 1.83(\mathrm{bs}, 4 \mathrm{H}) .{ }^{13} \mathrm{C} \mathrm{NMR}\left(75 \mathrm{MHz}, \mathrm{CDCl}_{3}\right): \delta=158.7,149.2,137.0,122.8$ 121.7, 59.6, 54.8. MS (ES) $m / z 138.2(\mathrm{M}+\mathrm{H})^{+}$. Anal. Calcd for $\mathrm{C}_{7} \mathrm{H}_{11} \mathrm{~N}_{3}: \mathrm{C}, 61.29 ; \mathrm{H}, 8.08 ; \mathrm{N}$, 30.63. Found C, 60.99; H, 8.11; N, 30.50. 